\title{
Aversão alimentar condicionada a Ipomoea carnea var. fistulosa em ovinos
}

\author{
Conditioned food aversion to Ipomoea carnea var. Fistulosa in sheep
}

\author{
Maria de Lourdes Adrien ${ }^{I}$ Dale GardnerII James PfisterII \\ Clairton Marcolongo-Pereira ${ }^{\mathrm{I}}$ Franklin Riet-Correa ${ }^{\mathrm{III}}$ Ana Lucia Schild ${ }^{\mathrm{I}}$
}

\section{RESUMO}

Quinze ovinos foram adaptados ao consumo de Ipomoea carnea por 36 dias. Posteriormente, foram divididos ao acaso em três grupos de cinco ovinos cada e avertidos com cloreto de lítio (LiCl) (Grupo 1) e Baccharis coridifolia (Grupo 2). O Grupo 3 permaneceu como controle. Os ovinos foram desafiados ao consumo da planta na pastagem e em baias individuais até o $74^{\circ}$ dia após o primeiro dia da aversão. Nos dias 14 e 19, o número de ovinos que consumiu I. carnea no Grupo 3 foi significativamente maior que o número de ovinos que consumiu a planta nos Grupos 1 e 2 ( $P=0,004$ e $P=0,0004$, respectivamente). No dia 24, o número de ovinos que consumiu I. carnea foi significativamente maior no Grupo 3 do que no Grupo 1 ( $P=0,004)$. A partir do desafio do dia 29, não houve diferença significativa $(P>0,05)$ no consumo da planta entre os três grupos. A quantidade de I. carnea consumida pelos ovinos do Grupo 3 durante o período do experimento no dia 7 após o primeiro dia da aversão foi significativamente maior do que o consumo das ovelhas dos Grupos 1 e 2 ( $P=0,0002$ e $P=0,01$, respectivamente). Nos desafios seguintes, não houve diferença na quantidade de I. carnea ingerida pelos ovinos. Na avaliação do comportamento dos ovinos na pastagem infestada por I. carnea, o tempo destinado ao consumo da planta foi de no máximo $2,4 \% \pm 1,6 \%$ do total de tempo de pastejo, não havendo diferença entre os três grupos. I. carnea nunca foi consumida avidamente pelos ovinos experimentais e a aversão incompleta induzida pelos dois agentes utilizados como aversores ( $\mathrm{LiCl}$ e B. coridifolia) sugere que não seriam eficientes para reduzir ou eliminar o consumo de I. carnea pelos ovinos.

Palavras-chave: aversão alimentar, swainsonina, plantas tóxicas, Baccharis coridifolia, ovinos, Ipomoea carnea.

\section{ABSTRACT}

Fifteen sheep were adapted to consume I. carnea for 36 days. Subsequently sheep were randomly divided into three groups of five sheep each. Group 1 was averted with LiCl, group 2 was averted with $\boldsymbol{B}$. coridifolia, and Group 3 was the control group. The sheep were periodically tested by exposing to I. carnea in the pasture and in individual pens up to the $74^{\text {th }}$ day after the first day of aversion. On the $14^{\text {th }}$ and $19^{\text {th }}$ days the number of sheep in Group 3 that consumed I. carnea was significantly higher than the number of sheep that consumed I. carnea in Groups 1 and 2 ( $P=0.004$ and $P=0.0004$, respectively). On day 24 the number of sheep that consumed I. carnea was significantly higher in Group 3 than Group $1(P=0.004)$. After the challenge on the $29^{\text {th }}$ day no significant difference $(P>0.05)$ was observed in the consumption of the plant among the three groups. On day 7 of the aversion period control sheep (group 3) consumed more I. carnea than did the sheep from Groups 1 and 2 ( $P=0.0002$ and $P=0.01$, respectively). After this period there was no difference in the amount of I. carnea ingested by the sheep in other individual challenges. The maximum time spent by the sheep grazing I. carnea was $2.4 \% \pm 1.6 \%$ of the total grazing time and no difference was observed among the groups. I. carnea was never consumed avidly by the experimental sheep, and some LiCl animals did not learn to avoid the plant on a consistent basis. Conditioning an incomplete aversion indicates that neither $\mathrm{LiCl}$ or $\boldsymbol{B}$. coridifolia will work with sheep in field settings to reduce or eliminate consumption of I. carnea.

Key words: food aversion, swainsonine, toxic plants, Baccharis coridifolia, sheep, Ipomoea carnea.

\section{INTRODUÇÃO}

Ipomoea carnea var. fistulosa (Convolvulaceae) é uma planta que contém swainsonina e produz intoxicação natural em caprinos (DE BALOGH et al., 1999; ARMIÉN

\footnotetext{
'Laboratório Regional de Diagnóstico, Faculdade de Veterinária (FVET), Universidade Federal de Pelotas (UFPel), Campus Universitário s/n, 96010-900, Pelotas, RS, Brasil. E-mail: alschild@terra.com.br. *Autor para correspondência.

IIUnited State Department of Agriculture (USDA), Agriculture Research Service (ARS), Poisonous Plant Research Laboratory, Utah State University (USU), Logan, UT, USA.

IIIHospital Veterinário, Centro de Saúde e Tecnologia Rural (CSTR), Universidade Federal de Campina Grande (UFCG), Patos, PB, Brasil. Recebido 31.05.13 Aprovado 28.08.13 Devolvido pelo autor 23.11.13 CR-2013-0767.R2
} 
et al., 2007; OLIVEIRA et al., 2009) e bovinos (ANTONIASSI et al., 2007). Em ovinos, a doença espontânea foi observada em dois animais com sinais clínicos característicos da intoxicação (TOKARNIA et al., 1960). A intoxicação por I. carnea foi reproduzida experimentalmente na espécie ovina com sinais clínicos caracterizados por perda de peso, depressão marcada, tremores musculares, déficit na propriocepção e morte (ARMIÉN et al., 2011).

A intoxicação por I. carnea é importante no norte e nordeste do Brasil em caprinos e diversos trabalhos têm relatado a ocorrência da doença nos Estados do Pernambuco, Bahia, Pará e Paraíba (ARMIÉN et al., 2007; OLIVEIRA et al., 2009; PIMENTEL et al., 2012). A intoxicação espontânea em ovinos tem sido relatada por produtores rurais em regiões próximas às margens do rio São Francisco, entre os Estados da Bahia e Pernambuco (RIETCORREA, 2012-informe verbal).

Para o controle da intoxicação em caprinos, diversos trabalhos têm sido desenvolvidos com o objetivo de promover a aversão condicionada a esta planta, utilizando-se cloreto de lítio ( $\mathrm{LiCl})$ (PIMENTEL et al., 2012; 2013) e Baccharis coridifolia (ADRIEN, 2013-informe verbal) como agentes aversivos.

Cloreto de lítio é o emético mais usado em estudos de aversão condicionada a plantas tóxicas em animais e tem sido eficiente na indução de aversão para o consumo de Delphinium barbeyi em vacas (OLSEN et al., 1989), Cercocarpus montanus e Amelanchier alnifolia em ovinos (BURRITT \& PROVENZA, 1990) e Amorimia rigida em caprinos (BARBOSA et al., 2008) e ovinos (PACÍFICO DA SILVA \& SOTO-BLANCO, 2010). Tem sido utilizado, também, para o controle da intoxicação por Turbina cordata em caprinos (PIMENTEL et al., 2012) e para o controle da intoxicação por I. carnea em caprinos, em períodos de chuva (PIMENTEL et al., 2012).

B. coridifolia foi utilizado com sucesso na indução de aversão condicionada ao milho em ovinos (ALMEIDA et al., 2009) e na indução de aversão a $\boldsymbol{I}$. carnea em caprinos (ADRIEN, 2013-informe verbal). B. coridifolia ocorre no Sul do Brasil e outros países da América do Sul, sendo conhecida desde a época da colonização espanhola nas Américas pela população nativa como uma planta tóxica indutora de aversão (COBO, 1653).

O objetivo deste trabalho foi avaliar o comportamento de ovinos frente à técnica de aversão alimentar condicionada para o controle do consumo de I. carnea utilizando $\mathrm{LiCl}$ e $\boldsymbol{B}$. coridifolia.

\section{MATERIAL E MÉTODOS}

Foram utilizados 15 ovinos da raça Corriedale de $28 \pm 2,5 \mathrm{~kg}$ de peso corporal (pc), fêmeas de 1,5 anos de idade, adquiridos em uma propriedade localizada no município de Herval, onde não havia $\boldsymbol{I}$. carnea. A planta utilizada em todo o experimento foi colhida no município de Monte Bonito/RS. Na área onde foi realizado o experimento, foram plantadas mudas de I. carnea provenientes do mesmo local.

Os ovinos foram adaptados ao consumo de I. carnea, já que, por não conhecerem a planta, não a consumiam. Para isso, 36 dias antes do início dos procedimentos de indução da aversão, os ovinos receberam, durante três dias consecutivos (dias 36-34), $1 \mathrm{~g}$ de folhas de $\mathbf{I}$. carnea secas e trituradas, misturadas na ração, administrada na quantidade de $1,5 \%$ do pc, para conhecerem o cheiro e o sabor da planta. Após essa adaptação inicial, os ovinos foram colocados em grupos de cinco animais escolhidos aleatoriamente a cada dia de adaptação, em uma área de $93 \mathrm{~m}^{2}$ com 0,8 plantas de I. carnea $\mathrm{m}^{-2}$ para pastoreio por 30 minutos, nos dias 33-27 e 18-16 antes do início da aversão. Nos dias 5-3, também antes do início da aversão, os ovinos pastorearam no mesmo local por uma hora. A adaptação foi realizada, também, em baias individuais, oferecendose $25 \mathrm{~g}$ de folhas frescas de I. carnea a cada ovino por 10 minutos nos dias 26-22, 20, 19, 15, 11, 7 e 6 antes do início da aversão. A adaptação foi realizada até que todos os ovinos consumissem qualquer quantidade de folhas frescas de I. carnea espontaneamente, tanto nas baias como na pastagem. Durante estes 36 dias, quando não estavam nos procedimentos de adaptação, os ovinos permaneciam em baias individuais e recebiam ração comercial para ovinos na dose de $1,5 \%$ do pc e $0,2 \mathrm{~kg}$ de feno de alfafa, e água a vontade.

Após a adaptação, os ovinos foram divididos ao acaso em três grupos de cinco indivíduos cada (Grupos 1, 2 e 3). No dia 1 da aversão, pela manhã, após jejum de aproximadamente 12 horas, a cada ovino, individualmente, foram oferecidos $25 \mathrm{~g}$ de folhas frescas de I. carnea durante 10 minutos. Imediatamente após o consumo de qualquer quantidade da planta ingerida, os ovinos do Grupo 1 receberam via sonda oro-gástrica, $170 \mathrm{mg} \mathrm{kg}^{-1} \mathrm{de} \mathrm{pc}$ de $\mathrm{LiCl}$ diluídos em um litro de água e os ovinos do Grupo 2 receberam $0,25 \mathrm{~g} \mathrm{~kg}^{-1}$ de pc de B. coridifolia seco, administrado por ingestão forçada. Para os ovinos do Grupo 3, foi oferecida a mesma quantidade de folhas frescas de I. carnea, porém os animais não foram avertidos. Esse procedimento foi repetido nos dias 2, 3 e 7, naqueles ovinos que ingeriram qualquer quantidade de $\mathbf{I}$. carnea. 
Nos dias 14, 19, 24, 29, 44, 59 e 74 após o $1^{\circ}$ dia da aversão, foram realizados testes nas baias (desafios nas baias), para identificar se os ovinos estavam ou não avertidos ao consumo de I. carnea. Para isso, foram oferecidas $25 \mathrm{~g}$ de folhas frescas de I. carnea a cada ovino, individualmente, por 10 minutos, pesando-se as folhas não consumidas.

Nos dias 10, 15, 20, 30, 45, 60 e 75, após o $11^{\circ}$ dia da aversão, foram realizados testes na pastagem invadida por I. carnea (desafios na pastagem) para avaliar o comportamento de cada ovino na área durante uma hora. A disponibilidade de forragem na área, composta por Cynodon dactylon e Eragrostis sp., era de $347,7 \pm 132,8 \mathrm{~kg}$ de matéria seca por hectare. Foi utilizada a metodologia de amostragem instantânea, registrandose a atividade individual dos ovinos a cada 50 segundos, considerando-se o tempo total de uma hora em cada desafio (ALTMANN, 1974). As atividades foram classificadas em: a) consumo de I. carnea; b) consumo de pastagem nativa; e c) outras atividades.

Para a determinação da concentração de swainsonina na planta utilizada no experimento, foram coletadas as folhas de 15 plantas de I. carnea da área infestada. As plantas secas em temperatura ambiente foram enviadas ao Poisonous Plants Research Laboratory- USDA- Logan UT, USA. Foram incubados durante toda a noite, à temperatura ambiente, $50 \mathrm{mg}$ de folhas de I. carnea secas e trituradas com $1,5 \mathrm{~mL}$ de ácido acético. Posteriormente, a mistura foi centrifugada e $50 \mu \mathrm{L}$ da solução foram misturadas com $950 \mu \mathrm{L}$ de hidróxido de amônio. Esta última solução foi analisada pelo método de cromatografia líquida e espectrofotometria de massa (LC-MS/MS). A concentração de swainsonina determinada nas amostras de $\mathbf{I}$. carnea foi $0,057 \pm 0.004 \%$.

$\mathrm{O}$ número de ovinos que consumiu $I$. carnea nos diferentes grupos foi comparado pelo teste exato de Fisher (Frequency procedure, SAS version 9.2). O consumo de I. carnea quando a planta foi oferecida nas baias foi analisado pelo modelo misto de medidas repetidas (Mixed procedure, SAS version 9.2), sendo as repetições os dias de desafio no experimento. A frequência de consumo de I. carnea na pastagem foi analisada pelo modelo generalizado linear misto (Glimmix procedure, SAS version 9.2). O nível de significância utilizado foi $5 \%$.

\section{RESULTADOS}

Desafio ao consumo de I. carnea nas baias

$\mathrm{O}$ número de ovinos que consumiu $\boldsymbol{I}$. carnea oferecida fresca nos cochos durante os desafios realizados nas baias é apresentado na figura $1 \mathrm{~A}$.
Nos dias 14 e 19, o número de ovinos que consumiu I. carnea no grupo controle foi significativamente maior que o número de ovinos que consumiu a planta nos grupos avertidos $(\mathrm{P}=0,004 \mathrm{e}$ $\mathrm{P}=0,0004$, respectivamente). No dia 24 , o número de ovinos que consumiu I. carnea foi significativamente maior no Grupo 3 do que no Grupo $1(\mathrm{P}=0,004)$. A partir do desafio do dia 29, não houve diferença significativa $(\mathrm{P}>0,05)$ no consumo da planta entre os três grupos

Com relação à quantidade de $\mathbf{I}$. carnea consumida pelos ovinos durante o período do experimento, observou-se que, no dia 7, após o primeiro dia da aversão, as ovelhas do Grupo 3 consumiram a planta em quantidade significativamente maior do que as ovelhas dos Grupos 1 e $2(\mathrm{P}=0,0002$ e $\mathrm{P}=0,01$, respectivamente) (Tabela 1 ). A partir do dia 14 , não houve diferença significativa $(\mathrm{P}>0,05)$ na quantidade de planta consumida entre os três grupos.

Desafio ao consumo de I. carnea na pastagem

Não houve diferença significativa $(\mathrm{P}>0,05)$ no número de ovelhas que consumiu I. carnea entre os três Grupos nos desafios realizados na pastagem. Os resultados são apresentados na figura 1B.

$\mathrm{Na}$ avaliação do comportamento dos ovinos na pastagem de I. carnea, a média de tempo de consumo da planta para o Grupo 1 foi $1,2 \% \pm 1,7 \%$, para o Grupo 2 foi $2,4 \% \pm 1,6 \%$ e para o Grupo 3 foi $1,7 \% \pm 2,2 \%$ do total do tempo de pastejo. A média de tempo de consumo de forragem para o Grupo 1 foi 93,9\% $0 \pm 7,8 \%$, para o Grupo 2 foi $94,4 \% \pm 6,3 \%$ e para o Grupo 3 foi $97,5 \% \pm 2,0 \%$ do total do tempo de pastoreio. A média de tempo para outras atividades realizadas pelos ovinos durante os desafios foi $5,5 \% \pm 9,1 \%$ para o Grupo $1,3,4 \% \pm 5,8 \%$ para o Grupo 2 e $0,6 \% \pm 0,84 \%$ para o Grupo 3. Nas três atividades, não houve diferença significativa entre os três grupos $(\mathrm{P}>0,05)$.

\section{DISCUSSÃO}

Os resultados do presente experimento demonstraram que tanto o $\mathrm{LiCl}$ quanto $\boldsymbol{B}$. coridifolia produziram aversão incompleta ao consumo de $\boldsymbol{I}$. carnea pelos ovinos nos desafios realizados nas baias ou na pastagem. Resultados similares foram obtidos com o $\mathrm{LiCl}$ em experimentos de aversão a Cercocarpus montanus em cordeiros (BURRITT \& PROVENZA, 1989), a Delphinium barbeyi em vacas (RALPHS \& OLSEN, 1990) e a Leucaena leucocephala em cabras (GÓRNIAK et al., 2008). Em outros trabalhos, utilizando B. coridifolia como agente aversor ao consumo de milho seco, foi observado que houve 


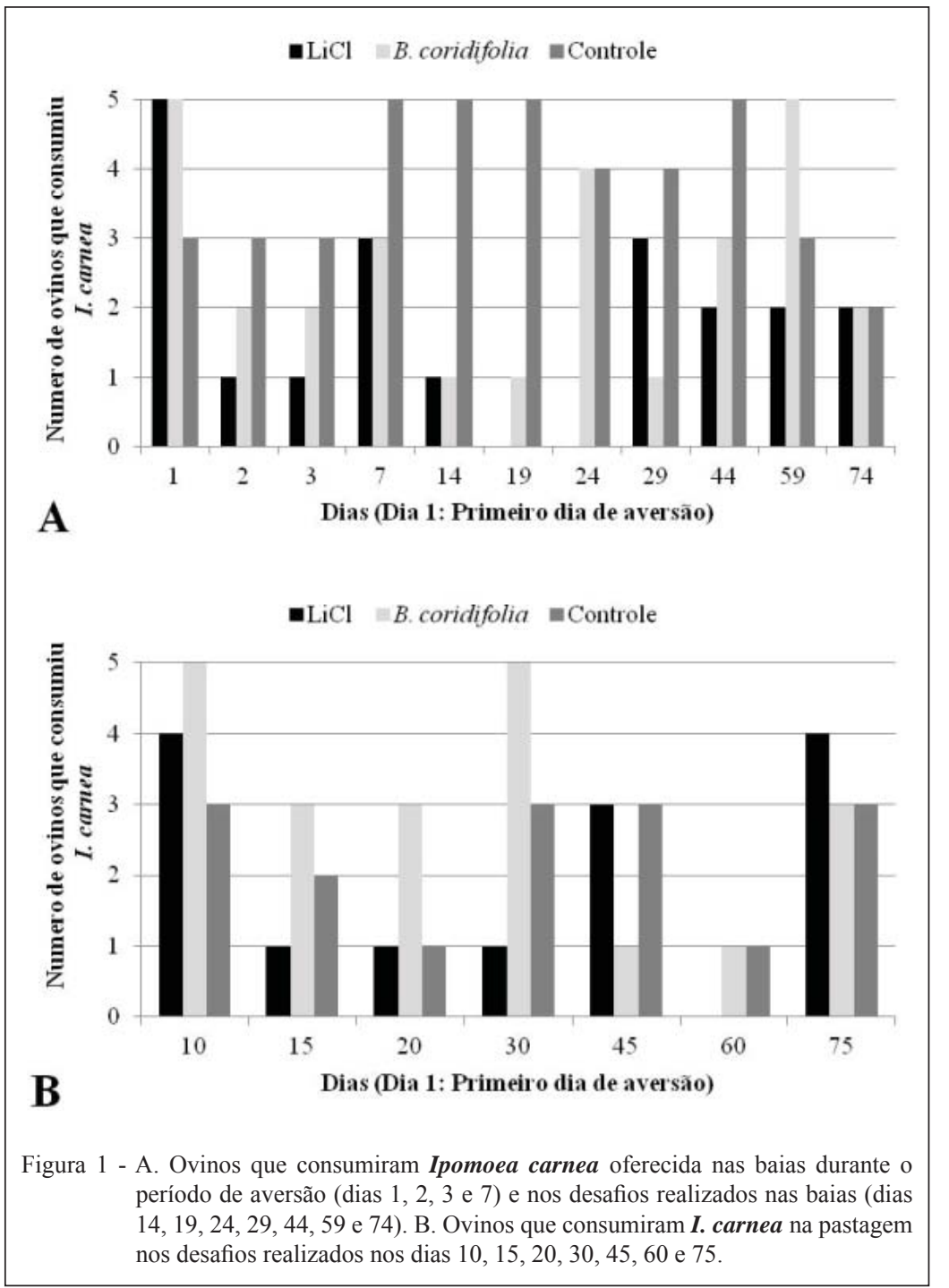

diminuição no consumo do grão durante o período de desafios (ALMEIDA et al., 2009).

No período de adaptação dos ovinos ao consumo de I. carnea, ficou evidenciado que esta espécie animal não consome a planta do mesmo modo que os caprinos. Nesta espécie, a intoxicação é frequente (ARMIÉN et al., 2007; OLIVEIRA et al., 2009; PIMENTEL et al., 2012). Por outro lado, apesar de os produtores das margens do Rio São Francisco mencionarem a ocorrência da intoxicação em ovinos, há apenas o registro da intoxicação em dois ovinos na Bahia (TOKARNIA et al., 1960), o que sugere que a doença é muito menos frequente nesta espécie do que em caprinos. Em experimentos realizados em cabras utilizando $\boldsymbol{B}$. coridifolia como agente aversor, foi demonstrado que, nessa espécie animal, esta planta é capaz de induzir aversão completa a I. carnea por pelo menos 330 dias (ADRIEN, 2013-informe verbal). A menor ocorrência da intoxicação em ovinos poderia ser, também, em consequência de que essa espécie não desenvolve preferência pelo consumo da planta como ocorre em caprinos (OLIVEIRA et al., 2009), além das diferenças nos hábitos alimentares entre estas duas espécies (PFISTER \& MALECHECK, 1986).

Durante todo o experimento, foi observado que o consumo de I. carnea era alternado e a quantidade consumida era variável, com tendência à diminuição em todos grupos, principalmente nos Grupos que foram avertidos, embora essa queda no consumo não tenha se mantido. $\mathrm{O}$ consumo com flutuações entre a ingestão e não ingestão de uma planta tem sido observado, também, em experimentos de comportamento dos animais frente ao consumo de plantas tóxicas, como o Delphinium barbeyi

Ciência Rural, v.44, n.2, fev, 2014. 
Tabela 1 - Consumo de folhas frescas de Ipomoea carnea $(\mathrm{g})$ nos dias da aversão e nos desafios realizados em baias individuais pelos ovinos avertidos com cloreto de lítio (Grupo 1), Baccharis coridifolia (Grupo 2) e controle (Grupo 3).

\begin{tabular}{|c|c|c|c|c|c|c|c|c|c|c|c|}
\hline \multirow{2}{*}{ Grupos } & \multicolumn{11}{|l|}{---- } \\
\hline & 1 & 2 & 3 & 7 & 14 & 19 & 24 & 29 & 44 & 59 & 74 \\
\hline Grupo 1 & $\begin{array}{l}23,5 \pm 2,4 \\
\mathrm{aA}\end{array}$ & $\begin{array}{l}11,1 \pm 5,4 \\
\mathrm{aB}\end{array}$ & $\begin{array}{l}12,4 \pm 5,4 \\
\mathrm{aB}\end{array}$ & $\begin{array}{l}4,1 \pm 3,1 \\
b B\end{array}$ & $\begin{array}{l}1,5 \pm 5,4 \\
\mathrm{aB}\end{array}$ & $\begin{array}{l}0,0 \\
a^{1}\end{array}$ & $\begin{array}{l}0,0 \\
a^{1}\end{array}$ & $\begin{array}{l}2,7 \pm 3,1 \\
\mathrm{aB}\end{array}$ & $\begin{array}{l}2,6 \pm 3,8 \\
\mathrm{aB}\end{array}$ & $\begin{array}{l}2,7 \pm 3,8 \\
\mathrm{aB}\end{array}$ & $\begin{array}{l}1,5 \pm 3,8 \\
\mathrm{aB}\end{array}$ \\
\hline Grupo 2 & $\begin{array}{l}21,3 \pm 2,4 \\
\mathrm{aA}\end{array}$ & $\begin{array}{l}5,4 \pm 3,8 \\
a B C\end{array}$ & $\begin{array}{l}5,8 \pm 3,8 \\
\mathrm{aBC}\end{array}$ & $\begin{array}{l}9,7 \pm 3,1 \\
b B C\end{array}$ & $\begin{array}{l}3,3 \pm 5,4 \\
\mathrm{aBC}\end{array}$ & $\begin{array}{l}5,0 \pm 5,4 \\
\mathrm{aBC}\end{array}$ & $\begin{array}{l}2,1 \pm 2,7 \\
a B C\end{array}$ & $\begin{array}{l}0,4 \pm 5,4 \\
\mathrm{aB}\end{array}$ & $\begin{array}{l}13,4 \pm 3,1 \\
\mathrm{aC}\end{array}$ & $\begin{array}{l}4,4 \pm 2,4 \\
\mathrm{aB}\end{array}$ & $\begin{array}{l}5,5 \pm 3,8 \\
\mathrm{aBC}\end{array}$ \\
\hline Grupo 3 & $\begin{array}{l}13,9 \pm 3,1 \\
\text { aACD }\end{array}$ & $\begin{array}{l}19,4 \pm 3,1 \\
\mathrm{aAD}\end{array}$ & $\begin{array}{l}14,2 \pm 3,1 \\
\mathrm{aAD}\end{array}$ & $\begin{array}{l}20,0 \pm 2,4 \\
\mathrm{aAD}\end{array}$ & $\begin{array}{l}2,5 \pm 2,4 \\
a B E\end{array}$ & $\begin{array}{l}3,0 \pm 2,4 \\
\text { aBE }\end{array}$ & $\begin{array}{l}4,5 \pm 2,7 \\
\text { aBEF }\end{array}$ & $\begin{array}{l}6,3 \pm 2,7 \\
\text { aCBGE }\end{array}$ & $\begin{array}{l}3,1 \pm 2,4 \\
\mathrm{aBH}\end{array}$ & $\begin{array}{l}11,0 \pm 3,1 \\
\text { aAFGH }\end{array}$ & $\begin{array}{l}9,8 \pm 3,8 \\
\mathrm{aEH}\end{array}$ \\
\hline
\end{tabular}

Letras minúsculas diferentes nas colunas indicam diferença significativa $(\mathrm{P}<0,05)$ no consumo de $\boldsymbol{I}$. carnea entre os grupos em cada desafio. Letras maiúsculas diferentes nas linhas indicam diferença significativa $(\mathrm{P}<0,05)$ no consumo de $\mathbf{I}$. carnea nos dias de desafio em cada grupo. Os ovinos foram avertidos nos dias $1,2,3$ e $7 ;{ }^{1}$ valores de $\mathrm{P}$ não estimados.

(PFISTER et al., 1997). Isso parece justificar a queda no consumo observada também no grupo controle deste experimento. Aparentemente, trata-se de um comportamento natural frente à ingestão de plantas tóxicas (FREELAND \& JANZEN, 1974).

Diferentes fatores podem influenciar na eficiência da aversão condicionada, independente do agente utilizado como aversivo. Dentre esses fatores, está o conhecimento prévio do alimento ao qual os animais serão avertidos (ROZIN \& KALAT, 1971). No presente estudo, o fato de que os ovinos foram adaptados por um período de mais de 30 dias poderia ter tornado I. carnea um alimento familiar, o que levou os animais a consumirem a planta, mesmo que em pequenas quantidades. Por outro lado, em caprinos adaptados ao consumo de I. carnea por mais de 30 dias, a aversão durou por pelo menos dois anos, no entanto, nos primeiros 30 dias, a adaptação foi com I. carnea seca e triturada, misturada na ração (RIET-CORREA, 2012-informe verbal).

Os resultados do presente estudo demonstraram que a aversão foi incompleta com os dois agentes utilizados como aversores $(\mathrm{LiCl}$ e $\boldsymbol{B}$. coridifolia), embora já tenha sido demonstrado que ambos são capazes de induzir aversão eficientemente em caprinos (PIMENTEL et al., 2012; 2013; ADRIÉN, 2013-informe verbal). Aparentemente, a aversão incompleta ocorreu em função de que $\boldsymbol{I}$. carnea não é uma planta consumida normalmente pelos ovinos.

\section{INFORME VERBAL}

Maria de Lourdes Adrien Delgado, 2013. Universidade Federal de Pelotas, Pelotas, RS, Brasil. E-mail: lourdesadrien@gmail.com.
Franklin Riet-Correa, 2012. Universidade Federal de Campina Grande, Patos, PB, Brasil. E-mail: franklin.riet@, pesquisador.cnpq.br.

\section{AGRADECIMENTOS}

Trabalho financiado pelo Institutos Nacionais de Ciência e Tecnologia (INCT)/ Conselho Nacional de Desenvolvimento Científico e Tecnológico (CNPq) (Proc.573534/2008-0), CNPq Proc. 471365/2010-6, CAPES Proc. 646/2010 (PROCAD-2009). Agradecemos ao Eng ${ }^{\circ}$ Agrônomo Oscar Bentancur, pelo auxílio na análise estatística.

\section{COMITÊ DE ÉTICA E BIOSSEGURANÇA}

Este estudo foi realizado entre abril de 2011 e maio de 2012, sendo aprovado pelo Comitê de ética e experimentação animal da Universidade Federal de Pelotas (UFPEL), Brasil (Protocolo número: 7775).

\section{REFERÊNCIAS}

ALMEIDA, M. et al. Conditioned aversion in sheep induced by Baccharis coridifolia. Applied Animal Behaviour Science, v.117, n.3-4, p.197-200, 2009. Disponível em: <http://naldc. nal.usda.gov/download/27125/PDF>. Acesso em: 10 abr. 2013. doi:10.1016/j.applanim.2008.12.006.

ALTMANN, J. Observational study of behaviour sampling methods. Behaviour, v.49, n.3-4, p.227-267, 1974. Disponível em: $<$ http://depts.washington.edu/cgfs/ifsp/Articles/Articles $\% 20$ for $\% 20$ Class/Observ-Study\%20Behav-methods-Behavior 1974. pdf>. Acesso em: 10 abr. 2013.

ANTONIASSI, N.A.B. et al. Intoxicação espontânea por Ipomoea carnea subsp. fistulosa (Convolvulaceae) em bovinos no Pantanal Matogrossense. Pesquisa Veterinária Brasileira, v.27, n.10, p.415-418, 2007. Disponível em: <http://www.scielo.br/pdf/pvb/ v29n7/14.pdf $>$. Acesso em: 10 abr. 2013.

ARMIÉN, A.G. et al. Clinical and morphologic changes in ewes and fetuses poisoned by Ipomoea carnea subspecies fistulosa. Journal of Veterinary Diagnostic Investigation, v.23, n.2, p.221-232. 2011. Disponível em: <http://vdi.sagepub.com/ 
content/23/2/221.full.pdf + html $>$. Acesso em: 10 abr. 2013. doi: $10.1177 / 104063871102300205$

ARMIÉN, A.G. et al. Spontaneous and experimental glycoprotein storage disease of goats induced by Ipomoea carnea subsp fistulosa (Convolvulaceae). Veterinary Pathology. v.44, n.2, p.170-184, 2007. Disponível em: $<$ http://vet.sagepub.com/content/44/2/170>. Acesso em: 10 abr. 2013. doi: 10.1354/vp.44-2-170.

BARBOSA, R.R. et al. Development of conditioned taste aversion to Mascagnia rigida in goats. Pesquisa Veterinária Brasileira, v.28, n.12, p.571-574. 2008. Disponível em: <http://www.scielo. br/pdf/pvb/v28n12/a01v2812.pdf >. Acesso em: 12 abr. 2013.

BURRITT, E.A.; PROVENZA, F.D. Food aversion learning: conditioning lambs to avoid palatable shrub (Cercocarpus montanus). Journal of Animal Science, v.67, p.650-653, 1989. Disponível em: <http://db.nr.usu.edu/qlc/pdf/R32108.pdf $>$. Acesso em: 10 abr. 2013.

BURRITT, E.A.; PROVENZA, F.D. Food aversion learning in sheep: persistence of conditioned taste aversions to palatable shrubs (Cercocarpus montanus and Amelanchier alnifolia). Journal of Animal Science, v.68, p.1003-1007, 1990. Disponível em: $\quad<$ http://www.journalofanimalscience.org/content/68/4/1003 full.pdf $>$. Acesso em: 10 abr. 2013.

COBO, B. Historia del nuevo mundo. España, Madrid: Biblioteca de Autores Españoles, [re-editado em 1964]. V.92, 1653. p.226-227.

de BALOGH, K.K.I.M. et al. A lysosomal storage disease induced by Ipomoea carnea in goats in Mozambique. Journal of Veterinary Diagnostic Investigation, v.11, p.266-273, 1999. Disponível em: <http://vdi.sagepub.com/content/11/3/266.long >. Acesso em: 10 abr. 2013.

FREELAND, W.J.; JANZEN, D.H. Strategies in herbivory by mammals: the role of plant secondary compounds. American Naturalist, v.108, n.961, p.269-289, 1974. Disponível em: $<$ http:// www.jstor.org/discover/10.2307/ 2459891? uid=2\&uid=4\&S $\mathrm{id}=21102017694121>$. Acesso em: 10 abr. 2013.

GÓRNIAK, S. et al. A note on averting goats to a toxic but palatable plant, Leucaena leucocephala. Applied Animal Behaviour Science, v.111, p.396-401, 2008. Disponível em: $<$ http://naldc.nal.usda.gov/download/15319/PDF>. Acesso em: 10 abr. 2013. doi:10.1016/j.Applanim.2007.06.005.

OLIVEIRA, C.A. et al. Poisoning by Ipomoea carnea subsp. fistulosa in goats in the Marajó island, Pará. Pesquisa Veterinária
Brasileira, v.29, n.7, p.583-588, 2009. Disponível em: <http:// www.scielo.br/pdf/pvb/v29n7/14.pdf $>$. Acesso em: 10 abr 2013.

OLSEN, J.D. et al. Aversion to eating poisonous larkspur plants induced in cattle by intraruminal infusion with lithium chloride. Journal of Animal Science, v.67, n.8. p.1980-1985,1989. Disponível em: <http:/www.journalofanimalscience.org/content/ 67/8/ 1980.full.pdf+html>. Acesso em: 10 abr. 2013.

PACÍFICO DA SILVA, I.; SOTO-BLANCO, B. Conditioning taste aversion to Mascagnia rigida (Malpighiaceae) in sheep. Research in Veterinary Science, v.88, n.2, p.239-241,2010. Disponível em: <http://www.sciencedirect.com/science/article/pii/ S0034528809002173>. Acesso em: 10 abr. 2013.

PFISTER, J.A. et al. Tall larkspur ingestion: can cattle regulate intake below toxic levels? Journal of Chemical Ecology, v.23, n.3, p.759-777, 1997. Disponível em: <http://link. springer.com/article/10.1023/B\%3AJOEC.0000006409.2027 9.59\#page-1>. Acesso em: 10 abr. 2013. doi. 10.1023/B:JO EC.0000006409.20279.59.

PFISTER, J.A.; MALECHECK, J.C. Dietary selection by goats and sheep in a deciduous woodland of Northeastern Brazil. Journal of Range Management, v.39, n.1, p.24-28, 1986. Disponível em:<http://www.jstor.org/discover/10.2307/3899680? uid $=2 \&$ uid $=4 \&$ Sid=21102017965501 $>$. Acesso em: 10 abr. 2013

PIMENTEL, L.A. et al. Aversão alimentar condicionada para o controle da intoxicação por Ipomoea carnea subsp. fistulosa em caprinos. Pesquisa Veterinária Brasileira, v.33, n.6. p.719-723, 2013. Disponível em: <http://www.pvb.com.br/pdf_artigos/04-07-2013_1546Vet\%201486_3187\%20LD.pdf>. Acesso em: 16 jul. 2013.

PIMENTEL, L.A. et al. Conditioned food aversion to control outbreaks of poisoning by Ipomoea carnea subsp. fistulosa and Turbina cordata in goats. Pesquisa Veterinária Brasileira, v.32, n.8. p.707-714, 2012. Disponível em: <http://dx.doi.org/10.1590/ S0100-736X2012000800005>. Acesso em: 10 abr. 2013.

RALPHS, M.H.; OLSEN, J.D. Adverse influence of social facilitation and learning context in training cattle to avoid eating larkspur. Journal of Animal Science, v.68, p.19441952,1990. Disponível em: <http://www.animal-science.org/ content/68/7/1944.full.pdf $>$. Acesso em: 10 abr. 2013.

ROZIN, P.; KALAT, J.W. Specific hungers and poison avoidance as adaptive specializations of learning. Psychological Review, v.78, n.6, p.459-486, 1971. Disponível em: <http://psycnet.apa. org/index.cfm?fa>. Acesso em: 10 abr. 2013.

TOKARNIA, C.H. et al. Estudo experimental sobre a toxidez do "canudo" (Ipomoea fistulosa Mart.) em ruminantes. Arquivos do Instituto Biológico Animal, v.3, p.57-71, 1960. 